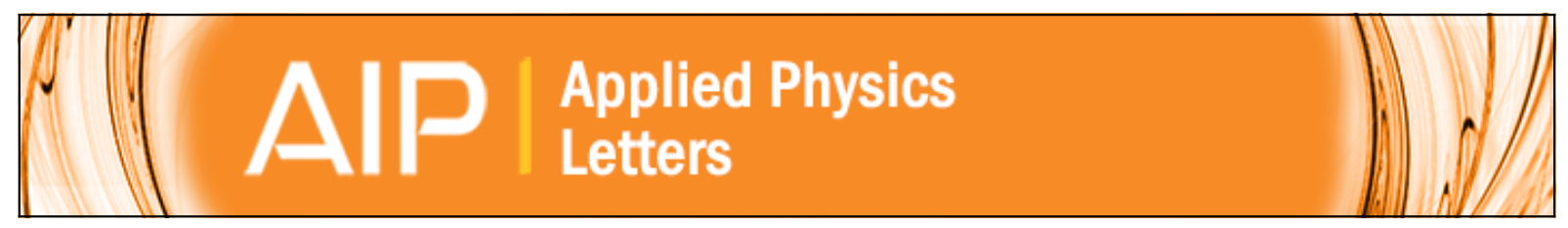

Alternating current loss in a cylinder with power-law current-voltage characteristic D.-X. Chen and C. Gu

Citation: Applied Physics Letters 86, 252504 (2005); doi: 10.1063/1.1947912

View online: http://dx.doi.org/10.1063/1.1947912

View Table of Contents: http://scitation.aip.org/content/aip/journal/apl/86/25?ver=pdfcov

Published by the AIP Publishing 


\title{
Alternating current loss in a cylinder with power-law current-voltage characteristic
}

\author{
D.-X. Chen ${ }^{\text {a) }}$ \\ ICREA and Grup d'Electromagnetisme, Departament de Física, Universitat Autònoma de Barcelona, 08193 \\ Bellaterra, Barcelona, Spain \\ C. $\mathrm{Gu}$ \\ Applied Superconductivity Research Center, Tsinghua University, Beijing 100084, China
}

(Received 4 January 2005; accepted 9 May 2005; published online 13 June 2005)

The transport ac loss $Q$ in a superconducting cylinder of radius $a$ with a power-law current-voltage characteristic $E=E_{c}\left|J / J_{c}\right|^{n}$ as a function of current amplitude $I_{m}$ is numerically calculated for a set of given values of $a, J_{c}$, and frequency $f$ at $n=5,10,20$, and 30. After deriving a scaling law and defining a critical frequency $f_{c}$, the results can be converted and interpolated to those for any values of $a, J_{c}, f$, and $5 \leqslant n \leqslant 30$. The "power-law" $Q\left(I_{m}\right)$ at $f=f_{c}$ is a better alternative of the critical-state $Q\left(I_{m}\right)$, used not only for describing the features of $Q\left(I_{m}, f\right)$ of high-temperature superconductors but also for planning $Q$ measurements and their interpretation. (C) 2005 American Institute of Physics.

[DOI: $10.1063 / 1.1947912]$

Soon after Bean assumed in the critical-state (CS) model a constant critical current density $J_{c}$ to calculate the magnetic property of a hard superconducting cylinder, ${ }^{1}$ London reported his derivation on the transport ac loss of a hard superconducting cylinder based on the same assumption. ${ }^{2}$ For a long cylinder of radius $a$, London gave the relation between the loss $Q$ per cycle per unit length and the amplitude of the transport ac current $I_{m}$ as

$$
\begin{aligned}
& q=\left(2-i_{m}\right) i_{m}+2\left(1-i_{m}\right) \ln \left(1-i_{m}\right), \\
& q \equiv 2 \pi Q / \mu_{0} I_{c}^{2}, \quad i_{m} \equiv I_{m} / I_{c},
\end{aligned}
$$

where $I_{c}=\pi a^{2} J_{c}$ is the critical current. The validity of this formula was later extended by Norris to an elliptical bar with arbitrary values of semiaxes $a$ and $b .^{3}$ In recent researches of high-temperature superconductors (HTSs), it has been shown that the measured $q$ versus $i_{m}$ curves of $\mathrm{Bi}-2223 / \mathrm{Ag}$ tapes is roughly consistent with Eq. (1), ${ }^{4-7}$ which means that the CS model is basically valid for such tapes. However, $J_{c}$ should be measuring time dependent owing to the flux creep mechanism of the actual CS. In the case of HTSs, the collective flux creep leads to a roughly power-law (PL) current-voltage $(I-V)$ characteristic, ${ }^{8-10}$

$$
\mathbf{E}=\left(E_{c} / J_{c}\right)\left|J / J_{c}\right|^{n-1} \mathbf{J}
$$

which is often shown in four-point measurements of HTSs. In Eq. (3), $J_{c}$ is routinely defined as the $J$ at which $E$ equals a critical value

$$
E_{c}=10^{-4} \mathrm{~V} / \mathrm{m} \text {. }
$$

With this PL $E(J), Q$ becomes a function of not only $I_{m}$, $J_{c}$, and $a$, such as in Eq. (1), but also $n$ and frequency $f$. In order to know the relation among these quantities, scaling laws have been studied by Rhyner and Sykulski et al. ${ }^{11,12}$ for the cases of infinite-semispace and infinite-slab bodies. Since essential parameters for long superconductors with a finite cross section are not considered, their results are hardly used by experimentalists. On the other hand, Brandt has stated a

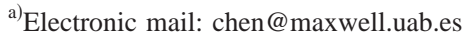

magnetic scaling law for any given sample with PL $E(J) .^{10}$ For cylindrical samples, Brandt's magnetic scaling law works for any given cylinder with a fixed radius $a$ but not for a general cylinder with a variable $a$, and therefore, his result cannot be simply used for a cylinder in the transport case. Thus, there has been an inconsistancy in the experimental study of HTS tapes; their $I-V$ curves are measured to be roughly of a PL type with a finite $n$ and an $I_{c}$ determined by the criterion Eq. (4), but their ac loss is compared with Eq. (1) that is derived from the CS model assuming $E=0$ when $|J|<J_{c^{*}}{ }^{4-7}$ In the present work, we will derive a transport scaling law for the cylinder with PL $E(J)$, so thataccompanied by a set of numerically calculated results-the above mentioned relation is quantitatively obtained and may be conveniently used in the ac loss research of HTS tapes.

We consider a current $I(t)=I_{m} \sin 2 \pi f t$ carrying superconducting cylinder of radius $a$ and infinite length along the $z$ axis. Disregarding the lower critical field, the constitutive laws of the superconducting material are

$$
\mathbf{B}=\mu_{0} \mathbf{H}, \quad \mathbf{J}=\sigma(J) \mathbf{E},
$$

where the conductivity is derived from Eq. (3) as

$$
\sigma(J)=\left(J_{c} / E_{c}\right)\left|J_{c} / J\right|^{n-1} .
$$

$Q$ can be obtained by solving a boundary and initial value problem of differential equation, which is derived as follows. Together with the Maxwell equations,

$$
\begin{aligned}
& \partial \mathbf{B}(\mathbf{r}, t) / \partial t=-\nabla \times \mathbf{E}(\mathbf{r}, t), \\
& \mathbf{J}(\mathbf{r}, t)=\nabla \times \mathbf{H}(\mathbf{r}, t),
\end{aligned}
$$

and using vector potential $\mathbf{A}$ defined by $\mathbf{B}=\nabla \times \mathbf{A}$ and $\nabla \cdot \mathbf{A}=0$, we obtain

$$
\nabla^{2} \mathbf{A}(\mathbf{r}, t)=\mu_{0} \sigma \partial \mathbf{A}(\mathbf{r}, t) / \partial t .
$$

In cylindrical coordinates according to the axial symmetry, $\mathbf{A}(\mathbf{r}, t)$ has a nonzero $z$ component $A(r, t)$ only, and when $r$ and $t$ are normalized as

$$
\rho=r / a, \quad \tau=2 \pi f t,
$$

Eqs. (6) and (9) lead to 
TABLE I. $q$ of a cylinder of $a=0.5 \mathrm{~mm}$ and $I_{c}=60 \mathrm{~A}$ as a function of $i_{m}$ and $n$, numerically calculated from Eq. (3) at $f=5 \mathrm{~Hz}$.

\begin{tabular}{lllll}
\hline \hline$i_{m}$ & $n=5$ & 10 & 20 & 30 \\
\hline 0.1 & 0.001368 & 0.000785 & 0.000546 & 0.000474 \\
0.1667 & 0.00563 & 0.00351 & 0.00257 & 0.00227 \\
0.333 & 0.0399 & 0.0279 & 0.0217 & 0.01963 \\
0.5 & 0.1302 & 0.0987 & 0.0801 & 0.0733 \\
0.667 & 0.308 & 0.255 & 0.215 & 0.1988 \\
0.833 & 0.599 & 0.559 & 0.500 & 0.470 \\
0.95 & 0.898 & 0.885 & 0.860 & 0.841 \\
1.0 & 1.065 & 1.090 & 1.101 & 1.102 \\
1.05 & 1.268 & 1.391 & 1.632 & 3.06 \\
1.1 & 1.517 & 1.858 & 2.97 & \\
1.2 & 2.19 & 3.68 & & \\
1.3 & 3.19 & 7.82 & & \\
\hline \hline
\end{tabular}

$$
\begin{aligned}
& \left|\left(\frac{1}{\rho} \frac{\partial}{\partial \rho}+\frac{\partial^{2}}{\partial \rho^{2}}\right) \frac{A(\rho, \tau)}{\mu_{0} I_{c}}\right|^{n-1}\left[\left(\frac{1}{\rho} \frac{\partial}{\partial \rho}+\frac{\partial^{2}}{\partial \rho^{2}}\right) \frac{A(\rho, \tau)}{\mu_{0} I_{c}}\right] \\
& \quad=\frac{2 \mu_{0} I_{c} f}{\pi^{n-1} E_{c}} \frac{\partial}{\partial \tau} \frac{A(\rho, \tau)}{\mu_{0} I_{c}} .
\end{aligned}
$$

Equation (11) should be solved under the boundary condition, which is obtained from the Ampere law, the A definition, and Eq. (5), and the initial condition

$$
\left.\frac{\partial}{\partial \rho} \frac{A(\rho, \tau)}{\mu_{0} I_{c}}\right|_{\rho=1}=-\frac{i_{m} \sin \tau}{2 \pi}, \quad \frac{A(\rho, 0)}{\mu_{0} I_{c}}=0 .
$$

After $t$ is increased from 0 to $m$ periods so that the symmetric periodical magnetization condition becomes stable, $q$ may be calculated as

$$
\begin{aligned}
q & =\frac{2 \pi}{\mu_{0} I_{c}^{2}} \int_{m / f}^{(m+1) / f} d t \int_{0}^{a} r J(r, t) E(r, t) d r \\
& =\frac{E_{c} \pi^{n}}{\mu_{0} f I_{c}} \int_{2 m \pi}^{2(m+1) \pi} d \tau \int_{0}^{1} \rho\left|\left(\frac{1}{\rho} \frac{\partial}{\partial \rho}+\frac{\partial^{2}}{\partial \rho^{2}}\right) \frac{A(\rho, \tau)}{\mu_{0} I_{c}}\right|^{1+n} d \rho,
\end{aligned}
$$

where Eqs. (3), (5), and (8), and the definition of $\mathbf{A}$ have been used.

From these equations, we see that under the stabilized current-sinusoidal condition and a fixed $n, A(\rho, \tau) / \mu_{0} I_{c}$ as a function of $i(\tau) \equiv I(\tau) / I_{c}$ is determined by $I_{c} f$ only. A scaling law can be stated as follows. If the value of $I_{c} f$ is multiplied by a constant $C$, the value of the resultant $A(\rho, \tau) / \mu_{0} I_{c}$ will be multiplied by $C^{1 /(n-1)}$, so that the values of $q$ and $i_{m}$ will be multiplied by $C^{2 /(n-1)}$ and $C^{1 /(n-1)}$, respectively. With the help of the scaling law, any functional transport ac loss of the superconducting cylinder may be obtained from the numerical results for a set of well chosen cases. Brandt proposed a very efficient numerical technique to calculate magnetic properties from the PL $E(J),{ }^{9,10}$ which was later used by Yazawa et al. ${ }^{13}$ and Rhyner ${ }^{14}$ for calculating transport properties. Following the procedure described in Ref. 13, we calculate accurately (with 200 elements and $2 \times 10^{5}$ time steps) $Q$ as a function of $I_{m}$ and $n$ for arbitrarily fixed $a=0.5 \mathrm{~mm}$, $I_{c}=60 \mathrm{~A}$, and $f=5 \mathrm{~Hz}$. The resultant $q$ as a function of $i_{m}$ for $n=5,10,20$, and 30 are given in Table I and plotted in Fig. 1(a). With these data and the scaling law, we may give different functions by choosing different variable parameters,
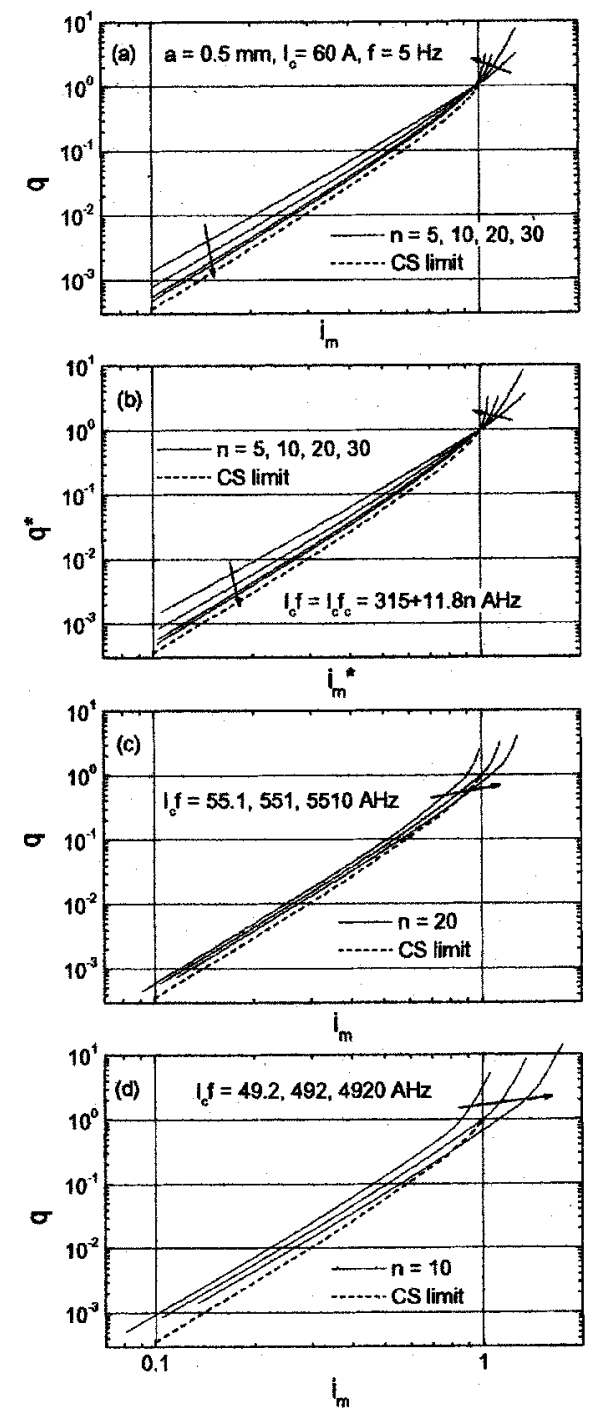

FIG. 1. (a) For a superconducting cylinder of radius $a=0.5 \mathrm{~mm}$ and critical current $I_{c}=60$ A with $E=E_{c}\left|J / J_{c}\right|^{n}$ characteristic of $n=5,10,20$, and 30, the normalized transport ac loss as a function of normalized current amplitude, $q\left(i_{m}\right)$, numerically calculated at $f=5 \mathrm{~Hz}$. (b) $q^{*}\left(i_{m}^{*}\right)$ curves scaled from $q\left(i_{m}\right)$ to critical frequency $f_{c}$. (c) and (d) $q\left(i_{m}\right)$ curves for $n=20$ and 10 at $f / f_{c}$ $=0.1,1$, and 10. The dashed lines are the CS limit $(n \rightarrow \infty)$ calculated from Eq. (1). Arrows indicate the direction of increasing $n$ or $f$.

but for the sake of clarity, we will fix the sample and temperature so that $I_{c}$ and $n$ are chosen as fixed parameters.

We first define a critical frequency $f_{c}$ at which the PL $q\left(i_{m}=1\right)=1$ occurs, so that any given $n$ corresponds to a unique and critical $q\left(i_{m}\right), q^{*}\left(i_{m}^{*}\right)$, which may be unambiguously compared with Eq. (1). The above numerical calculation is made at $I_{c}=60 \mathrm{~A}$ and $f=5 \mathrm{~Hz}$, and the resultant $q\left(i_{m}=1\right)>1$ for all values of $n$, as seen from Table I and Fig. 1(a). In order to meet the condition of $q^{*}\left(i_{m}^{*}=1\right)=1$ at any given $n$, we obtain $q^{*}\left(i_{m}^{*}\right)$ curve from the numerically calculated $q\left(i_{m}\right)$ at $f=5 \mathrm{~Hz}$ using the scaling law,

$$
i_{m}^{*}=i_{m} C^{1 /(n-1)}, \quad q^{*}=q C^{2 /(n-1)}, \quad f_{c}=C f,
$$

so that $f_{c}$ is obtained and approximately expressed by

$$
I_{c} f_{c}=315+11.8 n(\mathrm{~A} \mathrm{~Hz}, 5 \leqslant n \leqslant 30) .
$$

The $q^{*}\left(i_{m}^{*}\right)$ curves at $I_{c} f=I_{c} f_{c}$ are plotted in Fig. 1(b). We see that all PL curves cross over at $i_{m}^{*}=q^{*}=1$ so that they can be reasonably compared with the CS curve. When $i_{i m}^{*}<1, q^{*}$ increases with decreasing $n$ and the low $i_{m}^{*}$ limit of 


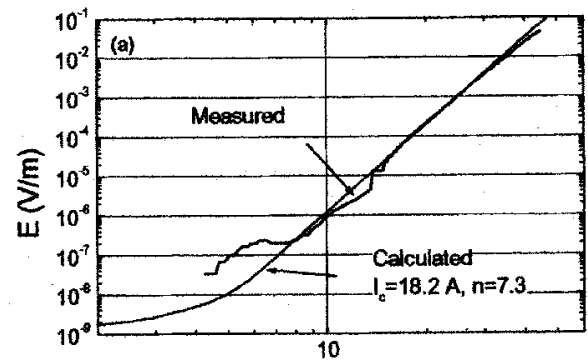

I(A)
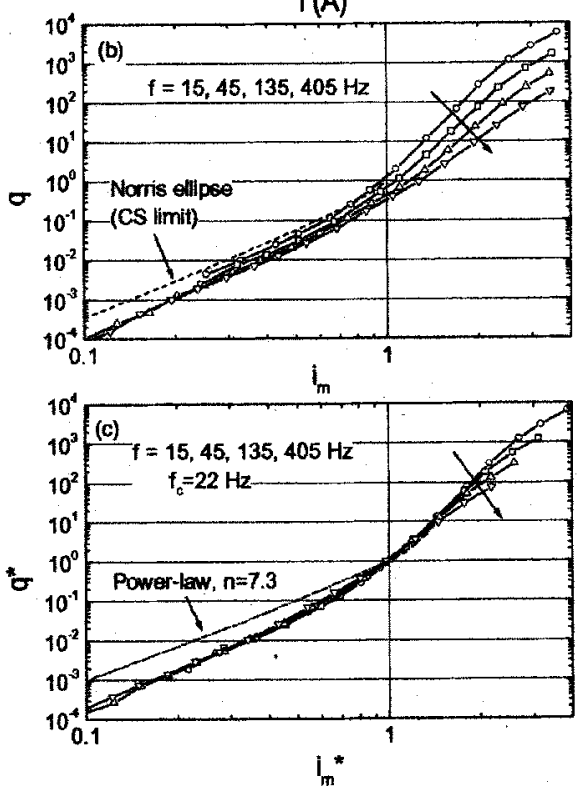

FIG. 2. (a) For a Bi-2223/Ag tape, the $E(I)$ curve measured at $77 \mathrm{~K}$ with current increasing rate $2 \mathrm{~A} / \mathrm{min}$, compared with the calculated one for an equivalent cylinder with $E=E_{c}\left|J / J_{c}\right|^{7.3}$ and $I_{c}=18.2 \mathrm{~A}$. (b) $q\left(i_{m}\right)$ measured at $77 \mathrm{~K}$ and $f=15,45,135$, and $405 \mathrm{~Hz}$. The dashed line is the CS limit calculated from Eq. (1). (c) $q^{*}\left(i_{m}^{*}\right)$ scaled from the measured $q\left(i_{m}\right)$ to $f_{c}$ $=22 \mathrm{~Hz}$. The dashed-dotted line is the PL $q^{*}\left(i_{m}^{*}\right)$ for $n=7.3$ calculated using Eqs. (14) and (15) and the data interpolated from Table I. The solid-line arrows in (b) and (c) indicate the direction of increasing $f$.

$d \lg q^{*} / d \lg i_{m}^{*}$ agrees well with $q^{*} \propto i_{m}^{* 3-2 /(n+1)}$, as previously predicted by Sykulski et al. ${ }^{12}$ for infinite slabs. When $i_{m}$ $>1$, the CS $q$ suddenly increases to very high values, whereas the PL $q^{*}\left(i_{m}^{*}\right)$ curve turns up continuously when $i_{m}^{*}$ passes unity and it turns more rapidly with increasing $n$.

We next investigate the frequency dependence of PL $q\left(i_{m}\right)$. In Figs. 1(c) and 1(d), we draw $q\left(i_{m}\right)$ at $f / f_{c}=0.1,1$, and 10 for $n=20$ and 10 , respectively. We see that the frequency dependence enhances with decreasing $n$. For each case, the entire $q\left(i_{m}\right)$ curve shifts to the upper-right-hand side when increasing $f$ from $f_{c}$, whereas it shifts to the lower left-hand side when decreasing $f$ from $f_{c}$. As a result, the curves at different values of $f$ are roughly parallel at small $i_{m}$. However, when $i_{m} \approx 1$, a decrease of $f$ below $f_{c}$ makes $q$ increase remarkably, especially when $n$ is large, although an increase of $f$ above $f_{c}$ decreases $q$ not as much.

Finally, we give an example to show how the derived scaling law can be used in the analysis of experimental results. The $E(I)$ curve of a multifilamentary Bi-2223/Ag tape made by the powder-in-tube technique is recorded from fourprobe measurement at $77 \mathrm{~K}$ as shown in Fig. 2(a), from which we see $I_{c}=18.2 \mathrm{~A}$ with a PL $E(I)$ dependence of $n$ $=7.3$ at $I \approx I_{c}$. The $q\left(i_{m}\right)$ measured at $77 \mathrm{~K}$ is plotted in Fig. 2(b) for $f=14,45,135$, and $405 \mathrm{~Hz}$. The $f_{c}$ is calculated using Eq. (15) to be $22 \mathrm{~Hz}$. Using the scaling Eq. (14), the four $q\left(i_{m}\right)$ curves for different values of $f$ are converted to $q^{*}\left(i_{m}^{*}\right)$ at $f_{c}=22 \mathrm{~Hz}$, as plotted in Fig. 2(c). We see that the original systematic $f$ dependent $q\left(i_{m}\right)$ curves become almost overlapped $q^{*}\left(i_{m}^{*}\right)$ curves when $i_{m}^{*}<1.5$. Some systematic difference among the four $q^{*}\left(i_{m}^{*}\right)$ curves may come from the departure of the measured $E(I)$ relation from the ideal $E(I)$ function calculated from the PL $E(J)$ with $n=7.3$, as shown by the dashed line in Fig. 1(a). The decrease of $q^{*}$ with increasing $f$ at $i_{m}^{*}>1.5$ may be related to the loss occuring in the normal metal Ag in the shell. ${ }^{15}$ The $q^{*}$ at $i_{m}^{*}<1$ to be lower than that calculated from the PL $E(J)$ and the CS $q$ calculated from Eq. (1) may come from a field dependent $J_{c}$, which is not considered in the derivation of Eq. (1) and the PL model presented above. ${ }^{16}$ Without going into more detail in the discussion, we can already see from this example that, in terms of $q^{*}\left(i_{m}^{*}\right)$ rather than Eq. (1), deeper qualitative and quantitative study on the ac loss of HTS tapes becomes possible.

In conclusion, we have derived a scaling law for the transport ac loss $Q$ per cycle per unit length of a superconducting cylinder with a PL $E(J)$ characteristic $E=E_{c}\left|J / J_{c}\right|^{n}$. After numerical calculation of $Q$ for some chosen cases, this scaling law enables us to obtain $Q$ as different functions of current amplitude $I_{m}$, frequency $f$, critical current $I_{c}$, cylinder radius $a$, and $n$. We have defined a critical frequency $f_{c}$ for any given cylinder and temperature with fixed $I_{c}$ and $n$ so that the normalized $Q$ as a function of the normalized $I_{m}, q\left(i_{m}\right)$, measured at any value of $f$ may be converted to a critical $q^{*}\left(i_{m}^{*}\right)$ function at $f_{c}$ and compared with the theoretical one calculated from the measured $I_{c}$ and $n$, leading to a deeper insight into the nature of the loss mechanism. Not containing sample size, the scaling law may be formally used for any given HTS tape and coated conductor.

The authors thank Spanish Ministerio de Educación y Ciencia Project No. FIS2004-02792 and Catalan projects SGR2001-00189 and CeRMAE for financial support.

${ }^{1}$ C. P. Bean, Phys. Rev. Lett. 8, 250 (1962).

${ }^{2}$ H. London, Phys. Lett. 6, 162 (1963).

${ }^{3}$ W. T. Norris, J. Phys. D 3, 489 (1970).

${ }^{4}$ Y. Yang, T. Hughes, C. Beduz, D. M. Spiller, R. G. Scurlock, and W. T. Norris, Physica C 256, 378 (1996).

${ }^{5}$ M. Majoros, B. A. Glowacki, A. M. Campbell, M. Apperley, and F. Garmann, Physica C 323, 125 (1999).

${ }^{6}$ J. Paasi, M. Masti, J. Lehtonen, and T. Kalliohaka, IEEE Trans. Appl. Supercond. 10, 1212 (2000).

${ }^{7}$ D.-X. Chen, X.-M. Luo, J.-G. Fang, and Z.-H. Han, Physica C 391, 75 (2003).

${ }^{8}$ G. Blatter, M. V. Feigel'man, V. B. Geshkenbein, A. I. Larkin, and V. M. Vinokur, Rev. Mod. Phys. 66, 1125 (1994).

${ }^{9}$ E.-H. Brandt, Phys. Rev. Lett. 76, 4030 (1996).

${ }^{10}$ E.-H. Brandt, Phys. Rev. B 58, 6506 (1998).

${ }^{11}$ J. Rhyner, Physica C 212, 292 (1993).

${ }^{12}$ J. K. Sykulski, R. L. Stoll, A. E. Mahdi, and C. P. Please, IEEE Trans. Magn. 33, 1568 (1997).

${ }^{13}$ T. Yazawa, J. J. Rabbars, B. ten Haken, H. H. J. ten Kate, and H. Maeda, J. Appl. Phys. 84, 5652 (1998).

${ }^{14}$ J. Rhyner, Physica C 310, 42 (1998).

${ }^{15}$ D.-X. Chen, E. Pardo, and A. Sanchez, Supercond. Sci. Technol. 17, 16 (2004).

${ }^{16}$ D.-X. Chen, A. Sanchez, and E. Pardo, Supercond. Sci. Technol. 17, 256 (2004). 